\title{
Simple circular dichroism method for selection of the optimal cyclodextrin for drug complexation
}

\author{
Eszter Kiss ${ }^{1}\left[\right.$ ] Virág Anna Szabó ${ }^{1} \cdot$ Péter Horváth $^{1}$ (i)
}

Received: 28 January 2019 / Accepted: 9 July 2019 / Published online: 15 July 2019

(c) The Author(s) 2019

\begin{abstract}
Cyclodextrins are very important excipients in the pharmaceutical industry. Given the multitude of native and semisynthetic cyclodextrin derivatives, there is a need for a rapid and reliable method for the selection of the optimal cyclodextrins for further pharmaceutical testing. During our research, circular dichroism (CD) spectroscopy has been successfully used to describe the qualitative and quantitative complexation of model compounds with different cyclodextrins. For the appearance of a circular dichroism signal, either a chiral or a chirally perturbed chromophore is required. Achiral or racemic compounds do not have corresponding circular dichroism spectra and neither do chiral cyclodextrins due to the absence of a chromophore group. During complexation of a chromophoric guest molecule, its absorption transition becomes chirally perturbed in the proximity of a cyclodextrin molecule and an induced circular dichroism (ICD) signal appears. This phenomenon gives an inherent selectivity to the method. The sign and intensity of the induced circular dichroism signal in case of different cyclodextrins provides information about the approximate structure of the complex as well as their stability relative to each other. In this study, we report a straightforward induced circular dichroism -based approach for the rapid preselection of the optimal cyclodextrin. The distinctive features of the method were demonstrated using five azole-type antifungal drug molecules (fluconazole, miconazole, clotrimazole, bifonazole and tioconazole) along with native $\alpha-, \beta$-, and $\gamma$-cyclodextrins, as well as dimethyl-, trimethyl-, carboxymethyl-, hydroxypropyl- and sulfobuthylether- $\beta$-cyclodextrins. In addition, with the aid of this method, 27 stability constants were determined, amongst which 16 have been unavailable in the literature previously.
\end{abstract}

Electronic supplementary material The online version of this article (https://doi.org/10.1007/s10847-019-00938-2) contains supplementary material, which is available to authorized users.

Eszter Kiss

kiss.eszter@pharma.semmelweis-univ.hu

1 Department of Pharmaceutical Chemistry, Faculty of Pharmacy, Semmelweis University, Hőgyes Endre u. 9, Budapest 1092, Hungary 


\section{Graphic abstract}
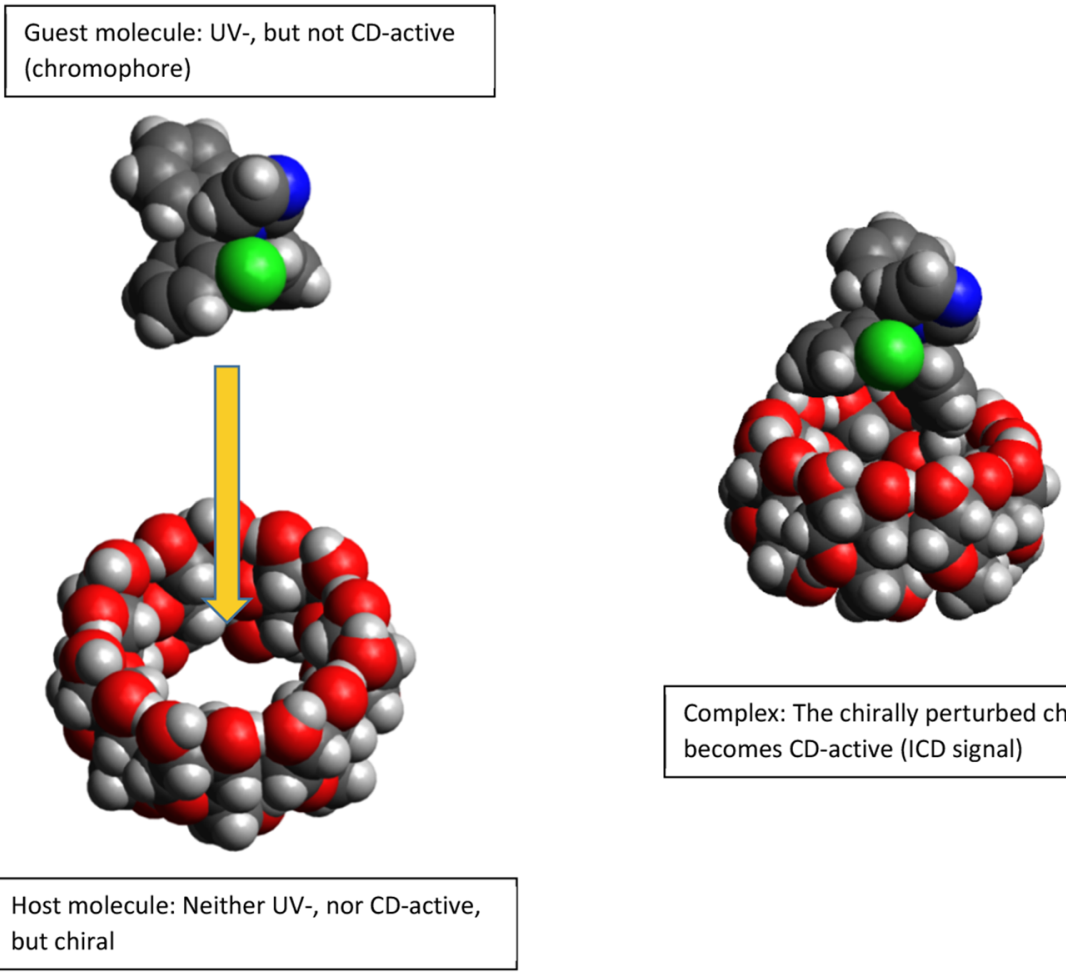

Complex: The chirally perturbed chromophore becomes CD-active (ICD signal)

Keywords Cyclodextrin $\cdot$ Host-guest complexation $\cdot$ Circular dichroism spectroscopy $\cdot$ Complex stability constant

$\begin{array}{ll}\text { Abbreviations } \\ \text { ACyD } & \alpha \text {-Cyclodextrin } \\ \text { BCyD } & \beta \text {-Cyclodextrin } \\ \text { BIZ } & \text { Bifonazole } \\ \text { CD } & \text { Circular dichroism } \\ \text { CE } & \text { Capillary electrophoresis } \\ \text { CLZ } & \text { Clotrimazole } \\ \text { CMBCyD } & \text { Carboxymethyl- } \beta \text {-cyclodextrin } \\ \text { DMBCyD } & \text { Dimethyl- } \beta \text {-cyclodextrin } \\ \text { DMSO } & \text { Dimethyl-sulfoxide } \\ \text { ESR } & \text { Electron spin resonance } \\ \text { FLZ } & \text { Fluconazole } \\ \text { GCyD } & \gamma \text {-Cyclodextrin } \\ \text { HPBCyD } & \text { Hydroxypropyl- } \beta \text {-cyclodextrin } \\ \text { HPLC } & \text { High performace liquid chromatography } \\ \text { ICD } & \text { Induced circular dichroism } \\ \text { IR } & \text { Infrared spectroscopy } \\ \text { MIZ } & \text { Miconazole } \\ \text { NMR } & \text { Nuclear magnetic resonance } \\ \text { NOESY } & \text { Nuclear overhauser effect spectroscopy } \\ \text { NP } & \text { Normal phase } \\ \text { PS } & \text { Phase solubility studies } \\ \text { PSA } & \text { Polar surface area }\end{array}$

$\begin{array}{ll}\text { ROESY } & \begin{array}{l}\text { Rotating frame nuclear overhauser effect } \\ \text { spectroscopy }\end{array} \\ \text { RP } & \text { Reverse phase } \\ \text { SBBCyD } & \text { Sulfobuthylether- } \beta \text {-cyclodextrin } \\ \text { TIZ } & \text { Thioconazole } \\ \text { TLC } & \text { Thin layer chromatography } \\ \text { TMBCyD } & \text { Trimethyl- } \beta \text {-cyclodextrin }\end{array}$

\section{Introduction}

Cyclodextrins are being used in numerous areas of the industry for a wide range of applications [1-4]. Their main property, the ability to form inclusion complexes can aid the dissolution of poorly soluble drugs, improve the retention of volatile molecules, and enhance chemical stability. However, despite having numerous advantages, cyclodextrins can cause some unexpected issues e.g.: in case of racemic drugs, cyclodextrins used in the pharmaceutical industry have different influence on the dissolution of enantiomers from the formulation $[5,6]$.

The selection of the most suitable cyclodextrin for a certain application can be made by using numerous analytical methods such as spectroscopic methods in solutions (UV 
spectrophotometry, NMR, fluorescence, ESR), separation techniques (CE, HPLC, TLC), and electroanalytical methods $[7,8]$. In case of solid phase samples, IR, Raman, X-ray, and thermal methods are the most commonly used, however, these approaches are not capable of determining the liquid phase (equilibrium) stability constant.

UV spectroscopy is a relatively straightforward method having low material needs, but it provides very little information about the structure of the complex. In this method, the change in the transitions of the chromophore group signals the formation of a complex [9]. As a result of the formation of an inclusion complex and the chromophore getting into a more apolar environment, the UV absorbance spectrum might become more structured than in an aqueous solution [10]. This interaction might result in either a hyper- or hypochromic and hypso- or bathocromic shift of the absorption spectrum. The two disadvantages of the method are that (a) other UV-active compounds may interfere with the spectrum, (b) the complexation does not necessarily result a characteristic change in the spectrum.

NMR spectroscopy has several advantages. Presence of a chromophore in the guest molecule is not required and, unlike in CD and UV spectroscopy, it provides information about the structure of the complex and it allows for quantification from the changes in the chemical shifts [11]. This method's drawback is its low sensitivity and the occasional need for deuterated solvents. If the guest molecule has low solubility, long acquisition times may be needed for achieving adequate signal-noise ratios in the case of 2D NOESY or ROESY techniques, which are necessary in structure analysis. The use of other solvents might be required to ensure the proper concentration of the compound. However, this additional solvent might affect the stability and the structure of the complex. In case of native cyclodextrins-due to their symmetry-the interpretation of the spectra can be simple, but in the case of modified cyclodextrins, the presence of structural isomers (different substitution degree) complicates the structural analysis.

The biggest advantage of fluorescence spectroscopy is the quantum yield effect, making it more sensitive and usable in a wider concentration range, compared to other techniques. It has to be noted that the signal intensity, despite being in correlation with the concentration, is not linear [12]. When using modified cyclodextrins containing fluorophore groups, the fluorescence spectrum changes due to interactions with the guest molecule. Commonly, the fluorophore is displaced from the cavity by the guest molecule in a competitive process. Based on this principle, cyclodextrins can be applied as molecular sensors [13].

Capillary electrophoresis requires small quantities of material, but either the guest molecule or the cyclodextrin must have a charged functional group. Neutral molecules and cyclodextrins cannot be tested directly. If the cyclodextrin is dissolved in the mobile phase, it might function as a chiral selector in case of enantiomer pairs [14].

HPLC methods are not restricted to charged molecules and apply cyclodextrins two ways: first, the cyclodextrin is covalently bonded to the solid phase [15]; second, the cyclodextrin is dissolved in the mobile phase [16].

When applying covalently bonded cyclodextrins, the separation can be performed using normal (NP) or reverse phase (RP) mode [17]. Testing different cyclodextrins require purchasing a column of each type. This is costly and time-consuming, especially if optimisation of the chromatographic parameters also requires addressing mobile phase composition and temperature parameters.

When cyclodextrins are dissolved in the mobile phase, a simple RP column (RP2-RP18) may be used to identify the optimal cyclodextrin by varying the cyclodextrin concentration and analysing the reduction in the retention time of the guest molecule. With this method, an apparent stability constant can be determined as well [18]. Problems posed by this technique are high costs, because for method optimisation large quantities of cyclodextrins might be required. In addition, optimization can be a lengthy procedure. Similarly, to capillary electrophoresis, attaining enantiomer selectivity can be set as an aim [19, 20].

Alongside the methods mentioned above, circular dichroism can come to mind as a good alternative. Its sensitivity and material needs are on par with UV spectroscopy but it provides more detailed structural information. Upon analysis of CD spectra, the formation of inclusion complexes can be confirmed and the structure of the complex can be assessed using theoretical calculations. Another benefit of this method is that, like UV spectroscopy, it can rapidly provide results, resulting in shorter experiment times. Furthermore, CD spectroscopy is less expensive than NMR or separation techniques.

The limiting factor of the method is the necessity of a chromophore on the guest molecule, which needs to be able to interact with the cyclodextrin to produce an induced CD signal (ICD) [21]. The appearance of this ICD signal in the region of the absorption bands is a definite proof of the complex formation. The sign and intensity of the ICD band, supplemented with theoretical calculations give opportunities for further estimations regarding the complex structure [22-24]. ICD spectra can be used to distinguish between cyclodextrins having different degrees of substitution [25]. In case of achiral or racemic substances when only the complex is CD active, it is possible to estimate the equilibrium (stability) constant, because the intensity of the ICD signal will be proportional to the complex concentration. Similar selectivity can only be achieved using NMR [26].

For the calculations, the following need to be taken into account: 
The equilibrium can be described with Eq. (1) in case of a 1:1 complex:

$$
\mathrm{K}_{\mathrm{e}}=\frac{[\mathrm{HG}]}{[\mathrm{H}]_{\mathrm{e}} \times[\mathrm{G}]_{\mathrm{e}}}
$$

where $\mathrm{K}_{\mathrm{e}}$ is the equilibrium constant, $[\mathrm{H}]_{\mathrm{e}}$ is the equilibrium concentration of the host, $[\mathrm{G}]_{\mathrm{e}}$ is the equilibrium concentration of the guest and $[\mathrm{HG}]$ is the concentration of the complex.

The equilibrium concentrations can be expressed as a function of the total concentrations as following

for the host: $[\mathrm{H}]_{\mathrm{e}}=[\mathrm{H}]_{\mathrm{T}}-[\mathrm{HG}]$

for the guest: $[\mathrm{G}]_{\mathrm{e}}=[\mathrm{G}]_{\mathrm{T}}-[\mathrm{HG}]$

where $[\mathrm{H}]_{\mathrm{T}}$ is the total concentration of the host and $[\mathrm{G}]_{\mathrm{T}}$ is the total concentration of the guest.

Replacing the equilibrium concentration of the host and the guest in Eq. (1) with the expressions from Eqs. (2) and (3), it can be written as:

$\mathrm{K}_{\mathrm{e}}=\frac{[\mathrm{HG}]}{\left([\mathrm{H}]_{\mathrm{T}}-[\mathrm{HG}]\right) \times\left([\mathrm{G}]_{\mathrm{T}}-[\mathrm{HG}]\right)}$

Expressing [HG] from Eq. (4) leads to a quadratic equation:

$[\mathrm{HG}]=\frac{[\mathrm{G}]_{\mathrm{T}}+[\mathrm{H}]_{\mathrm{T}}+\frac{1}{\mathrm{~K}}-\sqrt{\left([\mathrm{G}]_{\mathrm{T}}+[\mathrm{H}]_{\mathrm{T}}+\frac{1}{\mathrm{~K}}\right)^{2}-4[\mathrm{G}]_{\mathrm{T}}[\mathrm{H}]_{\mathrm{T}}}}{2}$

With the condition $[\mathrm{G}]_{\mathrm{T}} \geq[\mathrm{HG}] \geq 0$, the quadratic equation gives only the solution of Eq. (5) and the other roots does not need to be taken into consideration.

Keeping the concentration of the guest molecule constant and changing the concentration of the cyclodextrins one can obtain a saturation curve depending on the cyclodextrin excess and stability constant. The measured ellipticity $(\theta)$ correlates only with the concentration of the complex ([HG]) and at the maximum of the ellipticity signal true to Eq. (6):

$\theta_{\mathrm{MAX}} \approx[\mathrm{G}]_{\mathrm{T}}=[\mathrm{HG}]$

The stability constant can be calculated by using nonlinear parameter fitting for Eqs. (5) and (6)

$\frac{\theta}{\theta_{\mathrm{MAX}}}=\frac{[\mathrm{HG}]}{[\mathrm{G}]_{\mathrm{T}}} \rightarrow \theta=\frac{\theta_{\mathrm{MAX}}}{[\mathrm{G}]_{\mathrm{T}}} \times[\mathrm{HG}]$

Using these calculations, the stability constants of drug-cyclodextrin interactions can easily be compared and the most suitable cyclodextrin may be selected for the purpose in question. As a drawback, it needs to be mentioned that the acquired ICD signal has low intensity sometimes, resulting in disadvantageous signal-noise ratios in the case of low-stability complexes $(\log K<2)$. Light scattering can be another occurring problem. It can be detected from the UV spectra measured along the CD, outside of the absorption region, the increase in the baseline signals the presence of solid particles. While these can be removed from the solutions by filtration, accurate calculations cannot be made from the ellipticity data acquired. In racemic substances, the stability of the two diastereomer complexes are different, so the calculated data is only average or apparent constant.

To demonstrate the method's capability five antifungal azole molecules were tested with different cyclodextrins.

\section{Materials and methods}

The following materials are products of Cyclolab Ltd. (Budapest, Hungary): $\alpha$-cyclodextrin (ACyD); $\beta$-cyclodextrin (BCyD); $\gamma$-cyclodextrin (GCyD), heptakis(2,6-di-O-methyl)- $\beta$-cyclodextrin (DMBCyD); (2-hydroxypropyl)- $\beta$-cyclodextrin (HPBCyD) (D.S 5,5); heptakis(2,3,6-tri-O-methyl)- $\beta$-cyclodextrin (TMBCyD); $\beta$-cyclodextrin sulfobutyl ether sodium salt (SBBCyD) (D.S 6,3); carboxymethyl- $\beta$-cyclodextrin sodium salt (D.S 3,5) (CMBCyD); fluconazole [2-(2,4-difluorophenyl)1,3-bis(1H-1,2,4-triazol-1-yl)propan-2-ol], miconazole [(RS)-1-(2-(2,4-dichlorobenzyloxy)-2-(2,4-dichlorophenyl) ethyl)-1 $H$-imidazole] and bifonazole [ $R S)-1-[$ Phenyl(4phenylphenyl) methyl]-1H-imidazole] were pharmacopoeial grade (Ph. Eur. 7.8) products of Sandoz (Budapest, Hungary). Clotrimazole [1-[(2-chlorophenyl)- (diphenyl) methyl]-1H-imidazole] and tioconazole [(RS)-1-[2-[(2Chloro-3-thienyl)methoxy]-2-(2,4-dichlorophenyl)ethyl]$1 \mathrm{H}$-imidazole] as well as the dimethyl sulfoxide (DMSO) used to dissolve the drug were purchased from SigmaAldrich (Budapest, Hungary). For the structures of model compounds see Supplement Fig. S1. CD and UV experiments were performed on a Jasco J-720 spectropolarimeter (Jasco Ltd., Tokyo, Japan) in Jasco and Hellma cylindrical cuvettes with pathlengths ranging from 1 to $20 \mathrm{~mm}$. The nonlinear parameter fitting was performed with Origin 8.0.

Drugs were dissolved in DMSO to form $0.1 \mathrm{M}$ stock solutions, which were further diluted with distilled water to $0.0016 \mathrm{M}$ for the experiments. Cyclodextrin stock solutions were $0.016 \mathrm{M}$ in water. Experimental cyclodextrin (CyD)drug solutions were mixed with the following concentration ratios: $0: 1,0.5: 1,1: 1,2: 1,5: 1$ and 10:1. In certain occasions, 20:1 and 50:1 ratio were also prepared. To promote the solubility of the drugs, $5 \mu \mathrm{l} 2 \mathrm{M} \mathrm{HCl}$ was used ( $\mathrm{pH} \sim 2$ ).

First, solutions containing only the drugs were measured. For each experiment, the cuvette was chosen so the absorption at the absorption peak would not exceed 2. All the spectra were registered with the following parameters: scan speed: $50 \mathrm{~nm} / \mathrm{min}$; slit: $2 \mathrm{~nm}$; response (D.I.T.): $2 \mathrm{~s}$; accumulation 3-5 depending on the signal-noise ratio. 


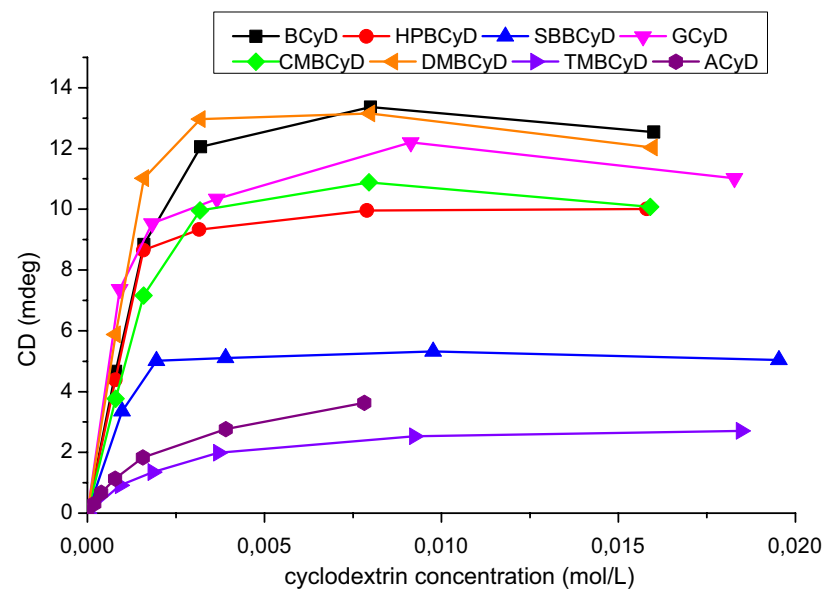

Fig. 1 Ellipticity of the BIZ:CyD complexes at increasing CyD concentrations $([\mathrm{BIZ}]=1.6 \mathrm{mmol} / \mathrm{L})$

\section{Results and discussion}

\section{Fluconazole}

Based on literature data, the $\log \mathrm{K}$ stability value of the complex changes depending on the protonation of the molecule. In case of $\beta$-cyclodextrin complexes, the $\log \mathrm{K}$ of the positively charged form is 1.03 , while for the unionised form it is 1.97 [27] or 1.83 [28]. At $\mathrm{pH} \sim 2$, the positively charged form is the dominant. Our previous, unpublished data showed 1.13 (positively charged) and a 1.64 (neutral) $\log \mathrm{K}$ for HPBCyD, 1.9 (charged) and 1.72 (neutral) for SBBCyD. During experiments ICD spectra could not be registered; see spectra in Supplement Fig. S2. Low $\operatorname{logK}$ values as well as low $\log P$ and high polar surface area (PSA) of FLZ is a possible explanation for this, based on previous literature [29] these two physical parameters, as well as the geometry of the molecule are the main factors affecting cyclodextrin stability constants.

\section{Bifonazole}

As a result of the intensive aromatic band at $\sim 250 \mathrm{~nm}$ of the biphenyl group in BIZ, the experiments were performed using $0.2 \mathrm{~cm}$ cuvettes as described in "Materials and methods" section. Figure 1 presents the measured CD signal at the highest ICD band as the function of CyD concentration. In case of $\mathrm{ACyD}$ at 5 and 10 time $\mathrm{CyD}$ excess precipitation occurred and the constant was calculated from fewer points. In case of $\mathrm{BCyD}, \mathrm{DMBCyD}, \mathrm{GCyD}$, and CMBCyD, at large $(10 \times)$ excess, the ellipticity values decreased. This signals the formation of a complex with a different stoichiometry in addition to $1: 1$. There is a possibility of the complexation of both the biphenyl and the phenyl group. Calculations were made without taking the last point(s) into consideration. In addition to changes in the $\mathrm{K}_{1: 1}$ ratio, the regression
Table 1 BIZ-CyD complex stability values and regression coefficients

\begin{tabular}{lll}
\hline CyD & $\begin{array}{l}\operatorname{LogK}_{1: 1}\left(\mathrm{M}^{-1}\right) \text { and } \\
\left(\mathrm{R}^{2}\right)(\text { all data })\end{array}$ & $\begin{array}{l}\operatorname{LogK}_{1: 1}\left(\mathrm{M}^{-1}\right) \text { and }\left(\mathrm{R}^{2}\right) \\
\text { (without the last data } \\
\text { point })\end{array}$ \\
\hline ACyD & $2.74(0.9966)$ & \\
TMBCyD & $2.95(0.9975)$ & $3.40(0.9964)$ \\
BCyD & $3.43(0.9898)$ & $3.40(0.9950)$ \\
CMBCyD & $3.59(0.9861)$ & $4.04(0.9636)$ \\
GCyD & $4.18(0.9565)$ & $4.21(0.9986)$ \\
DMBCyD & $4.39(0.9911)$ & \\
HPBCyD & $4.46(0.9957)$ & \\
SBBCyD & $4.72(0.9962)$ & \\
\hline
\end{tabular}

coefficient $\left(\mathrm{R}^{2}\right)$ also improved (Table 1). Constants for $\mathrm{BCyD}$ and CMBCyD became identical as can be expected from the slope of the curves in the first section.

Figure 2 shows the CD (Fig. 2a) and UV (Fig. 2b) spectra of BIZ, BIZ-BCyD, BIZ-SBBCyD and BIZ-GCyD complexes. While in the UV spectra of BIZ-BCyD and BIZ-SBBCyD a strong hypochromic and minimal hypsochromic effect can be observed compared to the spectrum of BIZ, BIZ-GCyD showed a bato- and hypochromic UV shift and a new smaller intensity CD band appeared at $235 \mathrm{~nm}$. Another difference in case of GCyD is the CD maximum appearing as higher wavelength (269) than in UV (258.4 nm), while BCyD and SBBCyD show a slight hypsochromic shift (255.6-254.6 nm). According to Table 1, the BIZ-SBBCyD complex has the highest stability value as a result of the electrostatic interaction between the protonated imidazole and negatively charged sulfobutyl groups. UV spectra of BCyD and SBBCyD at same cyclodextrin excess concentrations do not differ significantly, making it impossible to distinguish between the two; however, the difference in CD spectra proves their different complexation. In the case of SBBCyD the ionic interaction has influence on the depth of immersion and this fact results in significant difference between the BCyD and SBBCyD spectra. The GCyD shows notable bathochromic shift and relative low intensity. This fact allows to presume that the biphenyl chromophore is not parallel with the cyclodextrin's axis or there is space enough to immerse a phenyl group simultaneously.

\section{Clotrimazole}

PSA value of CLZ is similar to BIZ, however the $\log \mathrm{P}$ is higher as a result of the chlorophenyl group. Based on these parameters, $\mathrm{K}$ values are expected to be similar but the geometry of the molecule is different. CLZ is more compact, resulting in lower conformational freedom, also due to its molecular shape it is not expected to sink into the cavity like the biphenyl or phenyl group of BIZ. 

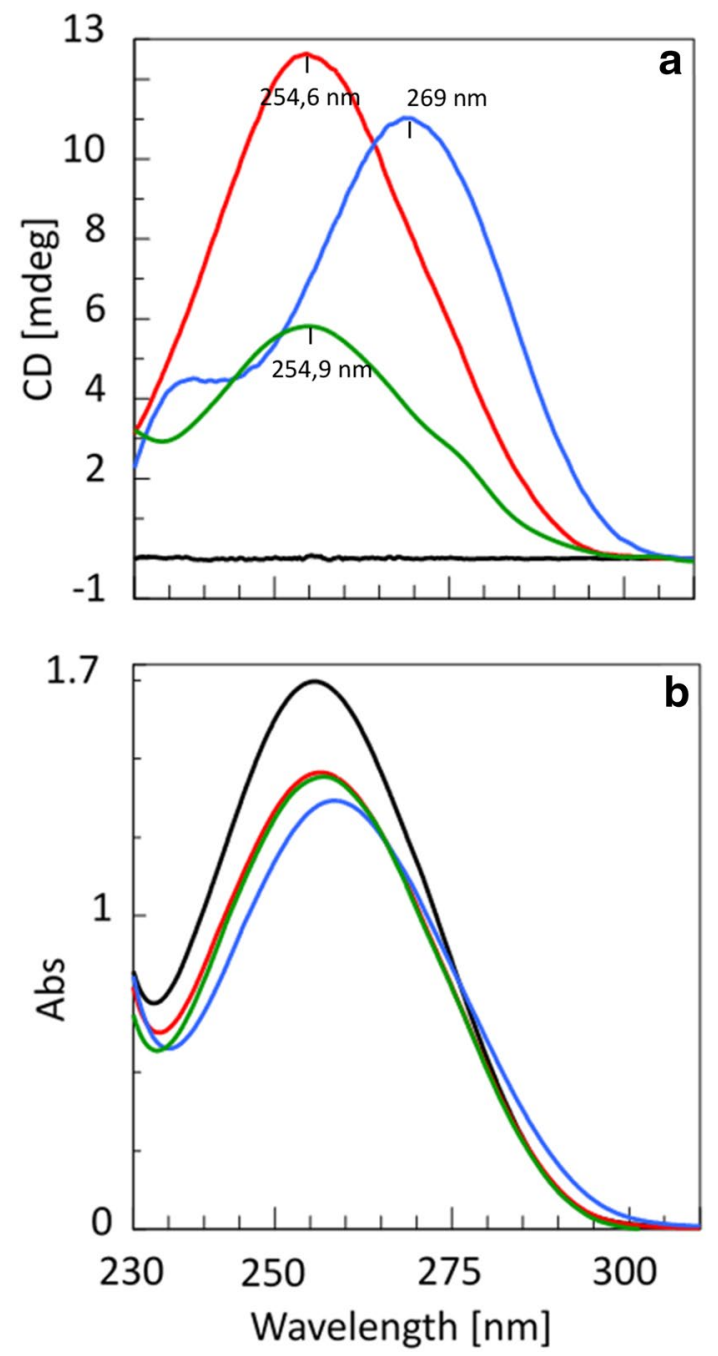

Fig. $2 \mathrm{CD}$ (a) and UV (b) spectra of BIZ (black), BIZ-BCyD (red), BIZ-GCyD (blue) and BIZ-SBBCyD (green) complexes $([\mathrm{BIZ}]=1.6 \mathrm{mmol} / \mathrm{L})$ The cyclodextrins are in $\times 5$ molar excess . (Color figure online)
In the case of CLZ, structural information cannot be gained from the UV spectra, neither the structural, neither intensity changes appear after CyD complexation; see Supplement Fig. S3. If observing simply the UV absorbance as a function of cyclodextrin concentration changes, the conclusion might be false, because there are no significant changes.

In contrast, $\mathrm{CD}$ spectra are highly informative. They can be divided into three groups (Fig. 3) based on their sign. Figure 3 a presents two structured spectra with dominantly negative ICD bands, but different intensity. Similarly, to BIZ, Fig. $3 \mathrm{a}$ also presents two positive ICD bands that are less structured than the negative bands on same figure and the alternating spectra of Fig. 3b, but are more informative than in case of BIZ. Figure $3 \mathrm{~b}$ also shows structured spectra, however, they contain alternating positive and negative bands. These characteristic changes-using Kodaka-Harata rules $[22,24]$-allow us to estimate inner-outer binding. Taking into consideration the length of the substituents and using the Kodaka-Harata rules, it can be assumed that the strong ionic interaction between the protonated imidazole ring and the negatively charged sulfobutyl side chain, does not allow the immersion of the molecule into the cavity. The shorter side chain (e.g. CMBCyD and HPBCyD) results in alternating positive-negative bands which means deeper immersion. $\mathrm{BCyD}$ and DMBCyD has only positive ICD bands because there is no steric inhibition.

Based on the ICD sign, intensity, and molecular geometry, it can be estimated that in case of SBBCyD-CLZ complex, CLZ is dominantly outside the cavity, only a small part of the molecule sinks in it. The sharply differentiated transitions assume a lower conformational freedom state. Outer binding is also supported by BCyD's cavity volume $\left(\sim 307 \AA^{3}\right)$ and CLZ's van der Waals volume $\left(\sim 309.52 \AA^{3}\right)$ being close numbers, thus the whole molecule is unable to fit into the cavity. This theory is supported by Pradines et al. [34]. Their NMR experiments and quantum chemical
Fig. 3 Different types of CLZ-CyD ICD spectra $([\mathrm{CLZ}]=1.6 \mathrm{mmol} / \mathrm{L})$. a RedTMBCyD, black-SBBCyD, orange-BCyD, aqua-DMBCyD. b Green-CMBCyD, blue-HPBCyD. The cyclodextrins are in $\times 10$ molar excess. (Color figure online)
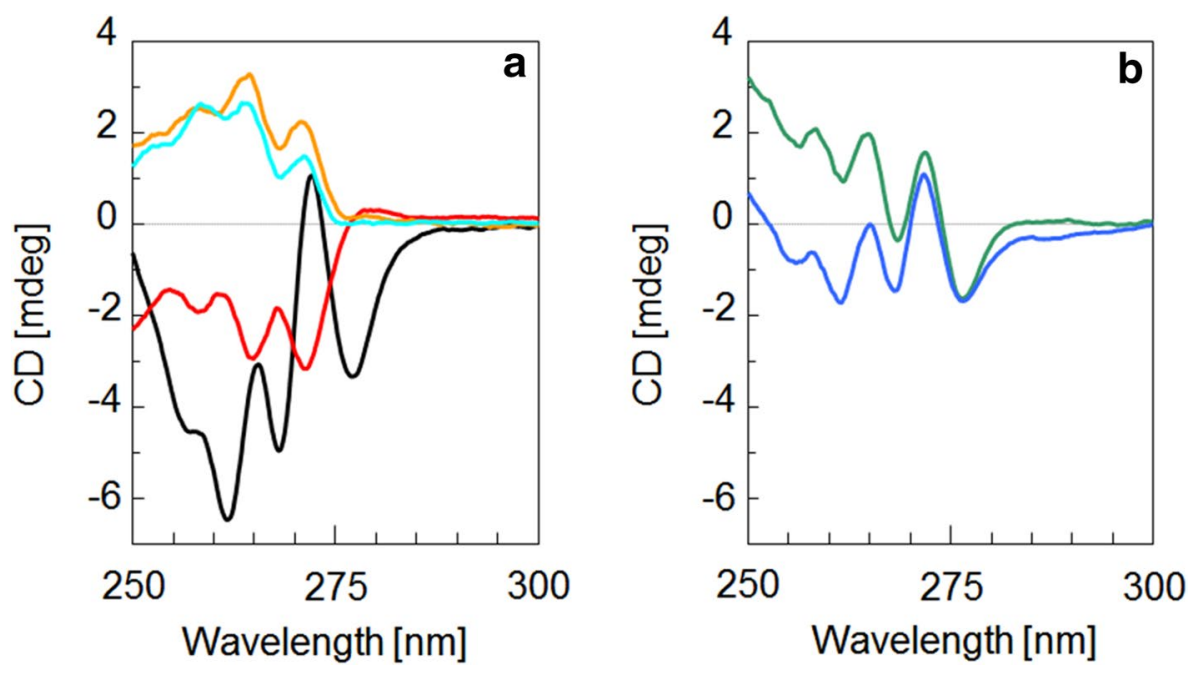
Table 2 CLZ-CyD complex-stability values and regression coefficients

\begin{tabular}{ll}
\hline CyD & $\operatorname{LogK}_{1: 1}\left(\mathrm{M}^{-1}\right)$ and $\mathrm{R}^{2}$ \\
\hline ACyD & - \\
GCyD & - \\
TMBCyD & $1.48(0.9996)$ \\
HPBCyD & $2.09(0.9966)$ \\
BCyD & $2.65(0.9861)$ \\
CMBCyD & $2.91(0.9802)$ \\
DMBCyD & $3.23(0.9924)$ \\
SBBCyD & $3.47(0.9972)$ \\
\hline
\end{tabular}

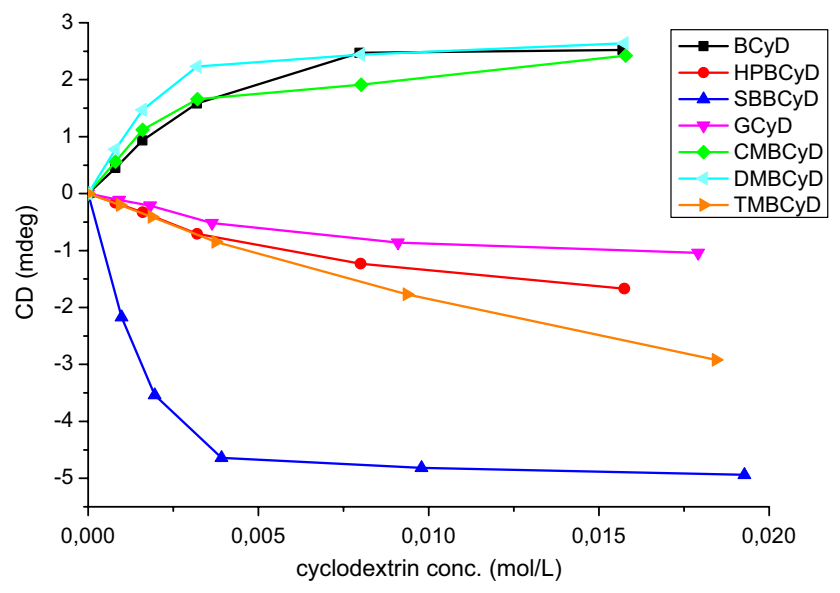

Fig. 4 Ellipticity of the CLZ:CyD complexes at increasing CyD concentrations $([\mathrm{CLZ}]=1.6 \mathrm{mmol} / \mathrm{L})$

calculations point out that only one substituent, the phenyl or chloro-phenyl moiety fits alternatively into the cavity of the cyclodextrin. This fact has to be taken into consideration with substituted $\beta$-cyclodextrins as well. As a result, calculated stability values in Table 2 are lower than BIZ's values. Due to precipitation, $\mathrm{ACyD}$ and GCyD constants could not be calculated. Figure 4 contains GCyD data only as briefs. Due to GCyD having a larger cavity, formation of inclusion complexes is possible, however, if during the excitation, the electrical dipole moment resultant is not parallel or perpendicular to the cyclodextrin's axis, the ICD sign becomes questionable. Because of precipitation, ACyD spectra could not be quantitatively evaluated, they showed a weak negative ICD signal. Since ACyD has an even smaller cavity than $\mathrm{BCyD}$, a weak outer biding is highly likely.

\section{Miconazole}

PSA value of MIZ (Table 3) is slightly higher than BIZ or CLZ, while the $\log P$ value is close to BIZ. Structural
Table 3 Molecular parameters and logarithm of stability constant $\left(\mathrm{M}^{-1}\right)$ of azole- $\beta$ CyD complexes

\begin{tabular}{llll}
\hline Compound & $\begin{array}{l}\text { Polar } \\
\text { surface area } \\
\left(\AA^{2}\right)^{\mathrm{a}}\end{array}$ & $\begin{array}{l}\log \mathrm{P}(\text { octanol/ } \\
\left.\mathrm{H}_{2} \mathrm{O}\right)\end{array}$ & $\log \mathrm{K}_{(1: 1)} \beta C y D C D$ \\
\hline Fluconazole & 81.65 & $0.41[30]$ & $1.03[24]$ \\
Miconazole & 27.05 & $4.50[31]$ & 2.70 \\
Tioconazole & 27.05 & $5.12[32]$ & 3.85 \\
Bifonazole & 17.82 & $4.77[32]$ & 3.40 \\
Clotrimazole & 17.82 & $5.20[32]$ & 2.65 \\
\hline
\end{tabular}

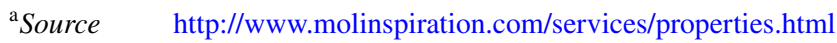
(16.01.2019) [33]
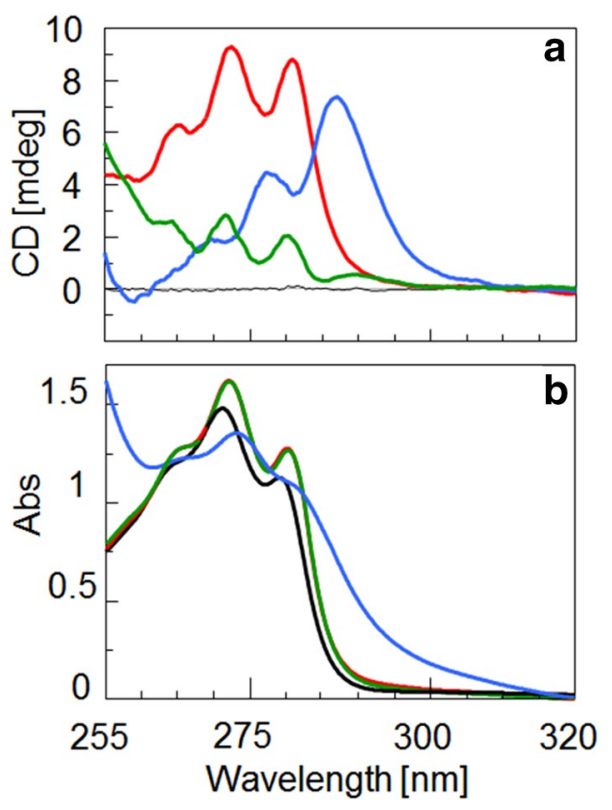

Fig. 5 CD (a) and UV (b) spectra of MIZ (black), MIZ-BCyD (red), MIZ-SBBCyD (green) and MIZ-GCyD (blue). The MIZ:CyD concentration ratio is $1: 10([\mathrm{MIZ}]=1.6 \mathrm{mmol} / \mathrm{L})$. (Color figure online $)$

features allow the molecule to have higher conformational freedom than either CLZ or BIZ. Based on molecular properties, the complex stability values are expected to be between the two molecules discussed earlier. The native UV spectrum maxima of miconazole-due to the dichlorophenyl substituent - appear at higher wavelengths in comparison with BIZ. The UV spectra of MIZ-CyD (Fig. 5b) complexes show a hyper- and batochromic shift compared to the original molecule and intensity changes are highly similar, making it impossible to distinguish between them with UV spectroscopy only. The only exception is GCyD, where the shift effects are stronger. Unlike on the previous occasions, characteristic benzoyl bands appear in the spectra. While the UV spectra of MIZ-BCyD and MIZ-SBBCyD almost fully overlap, their $C D$ spectra's intensity and shape show 


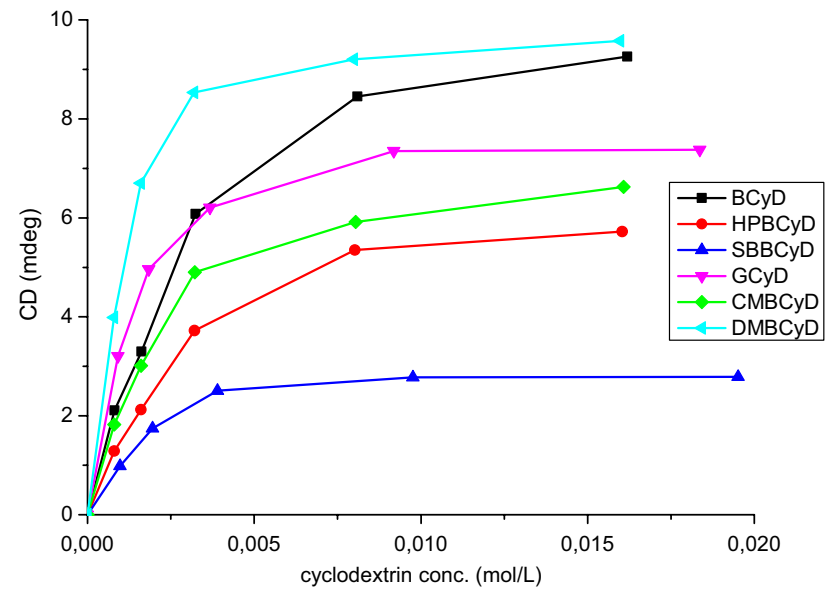

Fig. 6 Ellipticity of the MIZ:CyD complexes at increasing CyD concentrations $([\mathrm{MIZ}]=1.6 \mathrm{mmol} / \mathrm{L})$

Table 4 MIZ-CyD complex-stability values and regression coefficients

\begin{tabular}{ll}
\hline CyD & $\operatorname{LogK}_{1: 1}\left(\mathrm{M}^{-1}\right)$ and $\mathrm{R}^{2}$ \\
\hline BCyD & $2.70(0.9943)$ \\
HPBCyD & $2.71(0.9949)$ \\
CMBCyD & $2.96(0.9963)$ \\
SBBCyD & $3.18(0.9925)$ \\
GCyD & $3.37(0.9969)$ \\
DMBCyD & $3.68(0.9995)$ \\
\hline
\end{tabular}

significant differences (Fig. 5a). This difference may be in association with the immersion depth and the assumption based on the Kodaka-Harata rules [22, 24].

Figure 6 shows the ellipticity values read at the maximum of the most intensive transitions at increasing $\mathrm{CyD}$ concentrations; Table 4 presents the calculated stability values and regression coefficients. Most probably due to steric reasons, MIZ-SBBCyD was not the most stable, the constants acquired for both GCyD and DMBCyD complexes are higher. Higher conformational freedom and the size of the dichlorophenyl group can be listed among the steric reasons. At the beginning of the curves on Fig. 6, DMBCyD's ellipticity (cyan) changes the most, giving the highest stability value. It is followed by GCyD, having the second highest value. Based on our experience, the complex stability can be estimated from the slope of the curve's first part and equilibrium concentration, but not from the absolute intensity data.

\section{Tioconazole}

The structure of tioconazole resembles miconazole, the main difference being the replacement of the dichlorophenyl group
Table 5 TIZ-CyD complex-stability values and regression coefficients

\begin{tabular}{lc}
\hline CyD & $\operatorname{LogK}_{1: 1}\left(\mathrm{M}^{-1}\right)$ and $\mathrm{R}^{2}$ \\
\hline ACyD & $3.12(0.9877)$ \\
BCyD & $3.18(0.9925)$ \\
CMBCyD & $3.25(0.9362)$ \\
& $2.85^{\mathrm{a}}\left(0.9783^{\mathrm{a}}\right)$ \\
HPBCyD & $3.30(0.9998)$ \\
GCyD & $3.71(0.9921)$ \\
DMBCyD & $3.86(0.9997)$ \\
SBBCyD & $3.97(0.9996)$ \\
\hline
\end{tabular}

${ }^{\text {a }}$ Last two data points omitted

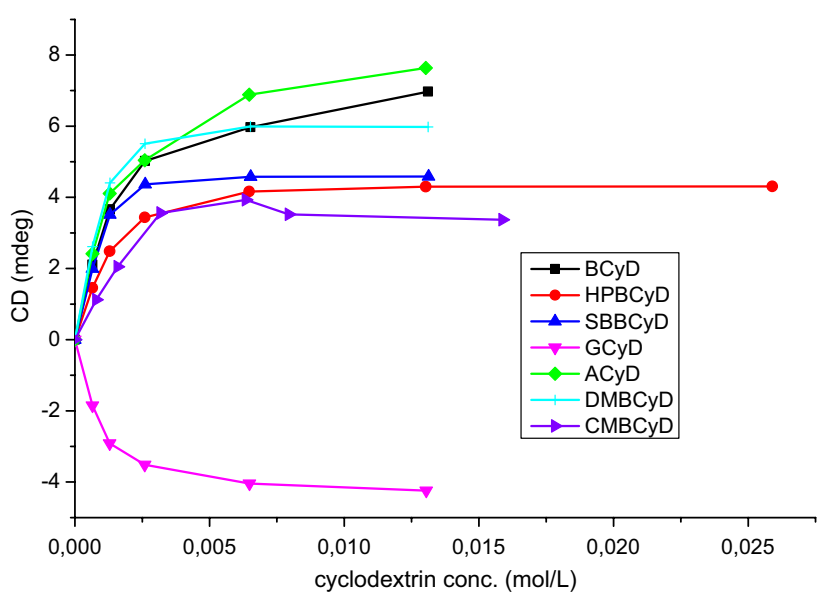

Fig. 7 Ellipticity of the TIZ:CyD complexes at increasing CyD concentrations $([\mathrm{TIZ}]=1.6 \mathrm{mmol} / \mathrm{L})$

with chlorothiophene. This change does not affect the PSA value but increases the lipophilicity slightly. The other difference is the size of the substituents; chlorothiophene is smaller than the dichlorophenyl group. The most intensive transition at the highest wavelength originates from the dichlorophenyl substituent as well as in case of MIZ. Comparing stability values, tioconazole's are higher (Table 5), the reason behind can be found in the structure and molecular parameters following from it. Despite the structural similarity, some differences can be found between MIZ- and TIZ-CyD complexes. First, the sign of the ellipticity of GCyD complexes: MIZ has a positive, while TIZ has a negative band, which suggests a different orientation of the chromophore in the complex (Fig. 7). In case of $\mathrm{CMBCyD}$, the ellipticity decreases at the highest cyclodextrin concentration, which proposes the formation of a complex with different stoichiometry than 1:1. Skipping the last two data points, the constant value decreases while $\mathrm{R}^{2}$ improves, however, the goodness of fit still does not reach the values calculated for the rest of the complexes. Tioconazole has a quite disadvantageous UV spectrum, it has only two 


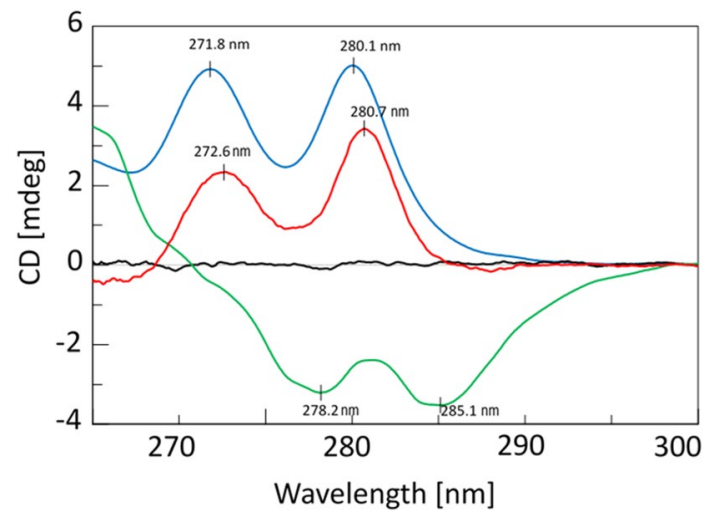

Fig. 8 CD spectra of TIZ (black), TIZ-ACyD (red), TIZ-HPBCyD (blue) and TIZ-GCyD (green) complexes at $\times 2$ molar excess of CyD-s $([T I Z]=1.6 \mathrm{mmol} / \mathrm{L})$. $($ Color figure online $)$

relatively flat peaks, one at 270.7 (shoulder only) the other at $279.5 \mathrm{~nm}$ (peak). Minor batochromic and hyperchromic shifts occur after cyclodextrin complexation, but the differences vary with the CyD used (see Supplement Fig. S4). ICD spectra always showed well-separated bands. These spectra can be classified into three groups (Fig. 8). ACyD and BCyD feature positive, closely equal intensity bands at both absorption regions. HPBCyD, DMBCyD, CMBCyD and SBBCyD are described by slight batochromic shift and increased intensity of the band at higher wavelengths, while GCyD showed strong batochromic shift and negative ICD bands (Fig. 8).

\section{Summary}

Circular dichroism is a suitable method for investigating the interaction of guest molecules with cyclodextrins. The appearance of the ICD signal is a matchless and strong proof of a non-covalent host-guest type inclusion complex formation. Such unambiguous, straightaway and fast evidence cannot be achieved by any other method. The intensity, sign, structure and shifts of spectral bands along with physicochemical properties and literature data allow for estimation of the complex structure. The CD spectroscopy may be an alternative to phase solubility studies also, because the UV and CD signals are measured in parallel, so the information about the increased solubility and the complexed guest concentration can be measured on two channels. A further possibility is the use of a third channel, if the CD spectropolarimeter is equipped with a fluorescent detector.

Taking into consideration that both the imidazole and triazole chromophores absorption maxima appear under $240 \mathrm{~nm}$, the signals must arise from the phenyl groups. The $\mathrm{L}_{B}$ band of a phenyl group have maximum about $250 \mathrm{~nm}$, which is highly affected by several factors e.g. solvents, substituents, and the homo- and hetero-molecular interactions. The fine structure of this transition is affected by the polarity of its surroundings and by conformational freedom. The complexation ensures a more apolar media in the cavity of the cyclodextrin for the lipophilic part(s) of the molecule; therefore the $\Delta \mathrm{G}$ (Gibbs free energy) and $\Delta \mathrm{S}$ (entropy) changes are advantageous in regard to complex formation. These facts allow to assume that the apolar phenyl or substituted phenyl chromophores take part dominantly in the complexation. In the case of MIZ and TIZ the higher absorption wavelengths are associated with the dichlorophenyl moieties.

The method is also useful for calculating stability constants based on simple equations. The simplicity of the calculation follows from the selectivity of the method, because only the complex gives signal, while other methods give the average signal of the different coexisting species (free and complexed particles). A very stable complex may be unfavourable from the point of view of drug release. Comparing the obtained values with each other, it may provide information on which cyclodextrins are suitable for complexation, however the value of the stability constant should not be the only basis for the decision.

Some other spectral properties may also informative, e.g. elevated baseline in both the CD- and UV-spectra signal the

Table 6 Summary and comparison of $\log \mathrm{K}$ values are calculated and collected from the literature

\begin{tabular}{|c|c|c|c|c|c|c|c|c|c|c|}
\hline & \multicolumn{2}{|c|}{ Fluconazole } & \multicolumn{2}{|c|}{ Bifonazole } & \multicolumn{2}{|c|}{ Clotrimazole } & \multicolumn{2}{|c|}{ Miconazole } & \multicolumn{2}{|c|}{ Tioconazole } \\
\hline & Meas & Lit & Meas & Lit & Meas & Lit & Meas & Lit & Meas & Lit \\
\hline $\mathrm{ACyD}$ & - & & 2.74 & - & - & - & - & & 3.12 & - \\
\hline BCyD & - & 1.03 [27] (NMR) & 3.40 & $3.34-3.52$ [39] (CE) & 2.65 & 2.66 [41] (PS) & 2.70 & 2.91 [41] (PS) & 3.18 & $3.12[43](\mathrm{CE})$ \\
\hline DMBCyD & - & & 4.21 & - & 3.23 & - & 3.68 & - & 3.86 & $3.84[43](\mathrm{CE})$ \\
\hline TMBCyD & - & & 2.95 & $3.13-3.26$ [39] (CE) & 1.48 & - & - & - & - & - \\
\hline HPBCyD & - & 1.13 [27] (NMR) & 4.46 & 3.66 [38] (NMR) & 2.09 & $2.65[40](\mathrm{PS})$ & 2.71 & $\begin{array}{l}2.56[42](\mathrm{PS}) \\
2.41[44](\mathrm{PS})\end{array}$ & 3.30 & $2.86[43](\mathrm{CE})$ \\
\hline SBBCyD & - & 1.90 [27] (NMR) & 4.72 & 3.94 [38] (NMR) & 3.47 & - & 3.18 & - & 3.97 & - \\
\hline CMBCyD & - & & 3.40 & - & 2.91 & - & 2.96 & - & 3.25 & - \\
\hline GCyD & - & & 4.04 & $6.45(1: 2)[38]$ (NMR) & - & 3.04 [37] (PS) & 3.37 & - & 3.71 & - \\
\hline
\end{tabular}


appearance of colloidal particles, so the method is capable of detecting poorly soluble complexes. It needs mentioning that our results are based on presuming the existence of only 1:1 stoichiometry as well as not taking into consideration the different stability of enantiomers in case of racemic substances. If more detailed complex structure is required, then the use of multiple methods can provide us with more accurate data $[35,36]$, however it was not the aim of this research. Table 6 summarizes the calculated $\log \mathrm{K}$ values and gives a comparison with data mined from the literature.

Acknowledgements Open access funding provided by Semmelweis University (SE). The authors thank András Váradi, Ph.D. (Columbia University) for language editing and proofreading.

Open Access This article is distributed under the terms of the Creative Commons Attribution 4.0 International License (http://creativeco mmons.org/licenses/by/4.0/), which permits unrestricted use, distribution, and reproduction in any medium, provided you give appropriate credit to the original author(s) and the source, provide a link to the Creative Commons license, and indicate if changes were made.

\section{References}

1. Szejtli, J.: Past, present and future of cyclodextrin research. Pure Appl. Chem. 76, 1825-1845 (2004)

2. Loftsson, T., Brewster, M.E.: Pharmaceutical applications of cyclodextrins. 1. Drug solubilization and stabilization. J. Pharm. Sci. 85(10), 1017-1025 (1996)

3. Del Valle, E.M.M.: Cyclodextrins and their uses: a review. Process Biochem. 39(9), 1033-1046 (2004)

4. Hedges, A.R.: Industrial applications of cyclodextrins. Chem. Rev. 98(5), 2035-2044 (1998)

5. Szegvári, D., Zelkó, R., Horváth, P., Gergely, A.: Calculation of cyclodextrin-mediated enantiomer ratio shifting of racem norgestrel in aqueous solutions. J. Inclusion Phenom. Macrocycl. Chem. 57(1-4), 169-172 (2007)

6. Szegvari, D., Zelko, R., Horvath, P., Gergely, A.: Tracking of enantioselective solubility of rac-norgestrel in the presence of cyclodextrin by a CD spectroscopic method. Chirality 18(2), 121-126 (2006)

7. Mura, P.: Analytical techniques for characterization of cyclodextrin complexes in aqueous solution: a review. J. Pharm. Biomed. Anal. 101, 238-250 (2014)

8. Li, S., Purdy, W.C.: Cyclodextrins and their applications in analytical chemistry. Chem. Rev. 92(6), 1457-1470 (1992)

9. Hamai, S.: Association of inclusion compounds of $\beta$-cyclodextrin in aqueous solution. Bull. Chem. Soc. Jpn 55, 2721-2729 (1982)

10. Köhler, G., Grabner, G., Klein, C.T., Marconi, G., Mayer, B., Monti, S., Rechthaler, K., Rotkiewicz, K., Viernstein, H., Wolschann, P.: Structure and spectroscopic properties of cyclodextrin inclusion complexes. J. Inclusion Phenom. Mol. Recognit. Chem. 25(1), 103-108 (1996)

11. Ejchart, A., Koźmiński, W.: NMR of cyclodextrins and their complexes. In: Dodziuk, H. (ed.) Cyclodextrins and Their Complexes: Chemistry, Analytical Methods, Applications, pp. 231-254. Wiley-VCH Verlag GmbH \& Co. KGaA, Weinheim (2006)

12. Pistolis, G., Malliaris, A.: Limitations on the determination of the stoichiometry and equilibrium constants of weak complexes by computer fitting methods: experimental verification. Chem. Phys. Lett. 310(5), 501-507 (1999)

13. Ueno, A.: Review: fluorescent cyclodextrins for molecule sensing. Supramol. Sci. 3(1), 31-36 (1996)

14. Scriba, G.K.E.: Cyclodextrins in capillary electrophoresis enantioseparations-recent developments and applications. J. Sep. Sci. 31(11), 1991-2011 (2008)

15. Wang, Y., Ng, S.C.: HPLC enantioseparation on cyclodextrinbased chiral stationary phases. In: Scriba, G.K.E. (ed.) Chiral Separations: Methods and Protocols, pp. 69-79. Humana Press, Totowa (2013)

16. Yu, L., Wang, S., Zeng, S.: Chiral mobile phase additives in HPLC enantioseparations. In: Scriba, G.K.E. (ed.) Chiral Separations: Methods and Protocols, pp. 221-231. Humana Press, Totowa (2013)

17. Armstrong, D.W.: Chiral stationary phases for high performance liquid chromatographic separation of enantiomers: a mini-review. J. Liq. Chromatogr. 7, 353-376 (1984)

18. Claude, B., Morin, P., Lafosse, M., Andre, P.: Evaluation of apparent formation constants of pentacyclic triterpene acids complexes with derivatized $\beta$ - and $\gamma$-cyclodextrins by reversed phase liquid chromatography. J. Chromatogr. A 1049(1), 37-42 (2004)

19. Lindner, W.: Recent development in HPLC enantioseparation - a selected review. Chromatographia 24(1), 97-107 (1987)

20. Váradi, A., Horváth, P., Kurtán, T., Mándi, A., Tóth, G., Gergely, A., Kökösi, J.: Synthesis and configurational assignment of 1,2-dihydroimidazo[5,1-b]quinazoline-3,9-diones: novel NMDA receptor antagonists. Tetrahedron 68(50), 10365-10371 (2012)

21. Gawroński, J., Grajewski, J.: The significance of induced circular dichroism. Org. Lett. 5(18), 3301-3303 (2003)

22. Harata, K., Uedaira, H.: The circular dichroism spectra of the $\beta$-cyclodextrin complex with naphthalene derivatives. Bull. Chem. Soc. Jpn 48(2), 375-378 (1975)

23. Kajtár, M., Horváth-Toró, C., Kuthi, É., Szejtli, J.: A simple rule for predicting circular dichroism induced in aromatic guests by cyclodextrin hosts in inclusion complexes. Acta Chim. Hung. 110(3), 327-355 (1982)

24. Kodaka, M.: Application of a general rule to induced circular dichroism of naphthalene derivatives complexed with cyclodextrins. J. Phys. Chem. A 102(42), 8101-8103 (1998)

25. Buvári-Barcza, Á., Kajtár, J., Szente, L., Barcza, L.: Hydroxypropyl- $\beta$-cyclodextrins: induced circular dichroism spectra of included phenolphthalein as a function of the degree of substitution. J. Chem. Soc. Perkin Trans. 2(4), 489-491 (1996)

26. Dodziuk, H., Danikiewicz, W., Grabner, G., Krois, D., Brinker, U.H., Bilewicz, R., Chmurski, K., Kunitake, M., Ohira, A.: Circular dichroism of cyclodextrin complexes. In: Dodziuk, H. (ed.) Cyclodextrins and Their Complexes. Wiley Online Books. WileyVCH Verlag GmbH \& Co. KGaA, Weinheim (2006)

27. Orgován, G., Kelemen, H., Noszál, B.: Protonation and $\beta$-cyclodextrin complex formation equilibria of fluconazole. J. Inclusion Phenom. Macrocycl. Chem. 84(3), 189-196 (2016)

28. Upadhyay, S.K., Kumar, G.: NMR and molecular modelling studies on the interaction of fluconazole with $\beta$-cyclodextrin. Chem. Centr. J. 3(1), 9 (2009)

29. Li, H., Sun, J., Wang, Y., Sui, X., Sun, L., Zhang, J., He, Z.: Structure-based in silico model profiles the binding constant of poorly soluble drugs with $\beta$-cyclodextrin. Eur. J. Pharm. Sci. 42(1), 55-64 (2011)

30. Alimuddin, M., Grant, D., Bulloch, D., Lee, N., Peacock, M., Dahl, R.: Determination of $\log$ D via automated microfluidic liquid-liquid extraction. J. Med. Chem. 51(16), 5140-5142 (2008)

31. British Pharmacopoeia safety data sheet: https://www.pharmacopo eia.com/Catalogue/Preview?uri=\%2Fcontent $\% 2$ Ffile\%2Fproducts \%2Fhealthandsafety\%2FCat_253_GB.pdf (22.01.2019) 
32. Avdeef, A.: Absorption and Drug Development: Solubility, Permeability, and Charge State, 2nd edn. Wiley, Hoboken (2012)

33. Ertl, P., Rohde, B., Selzer, P.: Fast calculation of molecular polar surface area as a sum of fragment-based contributions and its application to the prediction of drug transport properties. J. Med. Chem. 43(20), 3714-3717 (2000)

34. Pradines, B., Gallard, J., Iorga, B., Gueutin, C., Ponchel, G., Loiseau, P., Bouchemal, K.: The unexpected increase of clotrimazole apparent solubility using randomly methylated $\beta$-cyclodextrin. J. Mol. Recognit. 28(2), 96-102 (2015)

35. Daruházi, Á.E., Szente, L., Balogh, B., Mátyus, P., Béni, S., Takács, M., Gergely, A., Horváth, P., Szőke, É., Lemberkovics, É.: Utility of cyclodextrins in the formulation of genistein: Part 1. Preparation and physicochemical properties of genistein complexes with native cyclodextrins. J. Pharm. Biomed. Anal. 48(3), 636-640 (2008)

36. Tóth, G., Mohácsi, R., Rácz, Á., Rusu, A., Horváth, P., Szente, L., Béni, S., Noszál, B.: Equilibrium and structural characterization of ofloxacin-cyclodextrin complexation. J. Inclusion Phenom. Macrocycl. Chem. 77(1), 291-300 (2013)

37. Pedersen, M., Bjerregaard, S., Jacobsen, J., Mehlsen Sørensen, A.: A genuine clotrimazole $\gamma$-cyclodextrin inclusion complexisolation, antimycotic activity, toxicity and an unusual dissolution rate. Int. J. Pharm. 176(1), 121-131 (1998)

38. Hajnal, K., Csillag, A., Hancu, G., Blanka, S., Fülöp, I.E.V., Berța, L., Orgován, G.: Characterization of inclusion complexes between bifonazole and different cyclodextrins in solid and solution state. Maced. J. Chem. Chem. Eng. 36(1), 81-91 (2017)
39. Castro-Puyana, M., Crego, A.L., Marina, M.L., García-Ruiz, C.: Enantioselective separation of azole compounds by EKC. Reversal of migration order of enantiomers with $\mathrm{CD}$ concentration. Electrophoresis 28(15), 2667-2674 (2007)

40. Mohammed, N.N., Pandey, P., Khan, N.S., Elokely, K.M., Liu, H., Doerksen, R.J., Repka, M.A.: Clotrimazole-cyclodextrin based approach for the management and treatment of Candidiasis-a formulation and chemistry-based evaluation. Pharm. Dev. Technol. 21(5), 619-629 (2016)

41. Van Doorne, H., Bosch, E.H., Lerk, C.F.: Formation arid antimicrobial activity of complexes of $\beta$-cyclodextrin and some antimycotic imidazole derivatives. Pharmaceutisch Weekblad 10(2), 80-85 (1988)

42. Tenjarla, S., Puranajoti, P., Kasina, R., Mandal, T.: Preparation, characterization, evaluation of miconazole-cyclodextrin complexes for improved oral topical delivery. J. Pharm. Sci. 87(4), 425-429 (1998)

43. Penn, S.G., Bergstrom, E.T., Goodall, D.M., Loran, J.S.: Capillary electrophoresis with chiral selectors: optimization of separation and determination of thermodynamic parameters for binding of tioconazole enantiomers to cyclodextrins. Anal. Chem. 66(18), 2866-2873 (1994)

44. Loftsson, T., Brewster, M.E.: Cyclodextrins as functional excipients: methods to enhance complexation efficiency. J. Pharm. Sci. 101(9), 3019-3032 (2012)

Publisher's Note Springer Nature remains neutral with regard to jurisdictional claims in published maps and institutional affiliations. 